\title{
A Low Noise Preamplifier for HPGe Detectors with Auxiliary Output for Over Range Signal Spectroscopy
}

\author{
Alberto Pullia, Member, IEEE, Stefano Capra
}

\begin{abstract}
We present a low-noise Charge-Sensitive Preamplifier for HPGe detectors able to swiftly recover from saturation, featuring an auxiliary output signal used for highresolution pulse-height spectroscopy of the over-range signals. Using the auxiliary output for analyzing the signals larger than $\sim 15 \mathrm{MeV}$ (mostly charged particles and heavy ions) and the principal preamplifier output for analyzing all other signals (ionizing radiations and charged particles) a wide overall spectroscopic energy range of $5 \mathrm{keV}$ to $180 \mathrm{MeV}$ is obtained. A single Pole-Zero adjustment serves both the principal preamplifier channel as well as the auxiliary output channel. The energy resolution of the auxiliary channel is better than $0.1 \%$ typically. The Equivalent Noise Charge of the preamplifier is $\mathbf{1 1 5}$ electrons r.m.s., i.e. $\sim 0.8 \mathrm{keV}$ fwhm, using a BF862 as input transistor operated at room temperature while simulating the detector with a capacitance of $22 \mathrm{pF}$.
\end{abstract}

\section{INTRODUCTION}

A new generation of nuclear-physics experiments with radioactive ion beams is foreseen in the near future. In these experiments $4 \pi$ arrays of monolithic multi-electrode position sensitive germanium detectors will be used for spectroscopy and tracking of $\gamma$ rays [1]-[6]. In these experiments the electronics must comply with strict specifications. Besides having a low noise, it must provide a fast rise time, because the shape of the leading edge of the preamplifier signal is a key signature of the physical position of the $\gamma$-photon interaction point inside the detector [7]. A large dynamic range is also required for a class of experiments where giant-dipole resonances can produce $\gamma$ photons in the 20 $\mathrm{MeV}$ range. Another key requirement is a reduced dead time in the presence of a background of charged particles (pions, kaons) in the 10 to $100 \mathrm{MeV}$ energy range.

Advanced low-noise preamplifiers have been developed for these applications [8]-[10] able to swiftly recover from saturation. Moreover an original approach has been proposed which allows accurate estimate of the amplitude of large signals even when the preamplifier's output voltage gets saturated, as based on a Time-over-Threshold measurement technique [11]-[13]. This technique requires a precise

Manuscript received November 23, 2015.

A. Pullia is with the Department of Physics of the University of Milano, ITALY and INFN-Milano, ITALY (telephone: +39 02503-17735, e-mail: alberto.pullia@mi.infn.it).

S. Capra is with the Department of Physics of the University of Milano, and INFN-Milano, ITALY (e-mail: stefano.capra@unimi.it). measurement of the time width $\Delta \mathrm{T}$ of a suitable square pulse provided by a Schmitt trigger comparator. The amplitude of the large signal is then evaluated by putting into a proper mathematical equation $\Delta \mathrm{T}$ and a few other parameters derived from the sampled preamplifier waveform [14]-[16]. We propose here a new circuit and technique where the energy of the large signal is obtained in a much simpler way by processing a suitable auxiliary pulse through a precise PulseHeight Analyzer.

\section{EXPERIMENTAL RESULTS}

In Fig. 1 a picture of the realized hybrid circuit implementing the new technique is shown. It is built on an alumina substrate for optimization of the noise performance [17] and is installed in a shielded box located $20 \mathrm{~cm}$ apart from the detector.
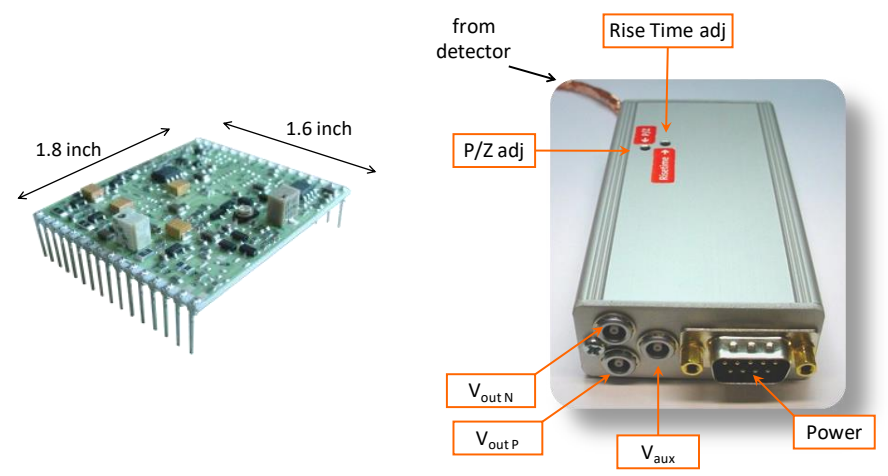

Fig. 1. Picture of the circuit board (left) and of the circuit as installed in its shielded box (right). The preamplifier output signal is differential, $\mathrm{V}_{\text {outP }}$ and $\mathrm{V}_{\text {out }}$ are the positive and negative signals. $\mathrm{V}_{\text {aux }}$ is a single-ended auxiliary signal used for spectroscopy of the large saturated events. A 10-turn trimmer allows precise Pole-Zero cancellation, which turns the ms long decay time of the detector into a shorter preamplifier decay time of $\sim 50 \mu \mathrm{s}$.

In Fig. 2 the experimental signals observed on the scope are shown for large equivalent events in the range from $10 \mathrm{MeV}$ to $100 \mathrm{MeV}$ with $10 \mathrm{MeV}$ step. Event (1) is under threshold and, as expected, $V_{\text {out }}$ is an exponential decay function. Events (2)(10), instead, yield saturation of $\mathrm{V}_{\text {out }}$. In these cases a fast-reset cycle gets triggered which turns the exponential decay function into a narrow trapezoid. As shown in the middle of 
Fig. 2 when a normal spectroscopy amplifier (shaper) is connected to $\mathrm{V}_{\text {out }}$ the amplitude of the shaped signal $\mathrm{V}_{\text {sh }}$ does not grow with the event energy because of saturation of its input signal, and no pulse-height spectroscopy is therefore possible. In order to retrieve the amplitude information the Time-over-Threshold scheme could be used, as mentioned in [11], with a price to be paid in terms of complexity. However a completely different behaviour is observed looking at the output of the auxiliary circuit, as shown in the top of Fig. 2. Now the pulse height nicely grows with the event energy for all considered cases, which allows spectroscopy of the large signals by simply using a precise Pulse-Height Analyzer. Note that for lower signals $<10 \mathrm{MeV}$ the amplitude of $\mathrm{V}_{\text {aux }}$ becomes too little for high-resolution spectroscopy. For these cases signals $\mathrm{V}_{\text {out }}$ or $\mathrm{V}_{\text {sh }}$ must instead be used. Signal processing in these cases is performed by using a digital filter or an analog shaper amplifier. The structure and all details of the circuit used to generate pulses " $V_{\text {aux }}$ "of Fig. 2 will be shown elsewhere in a dedicated paper.

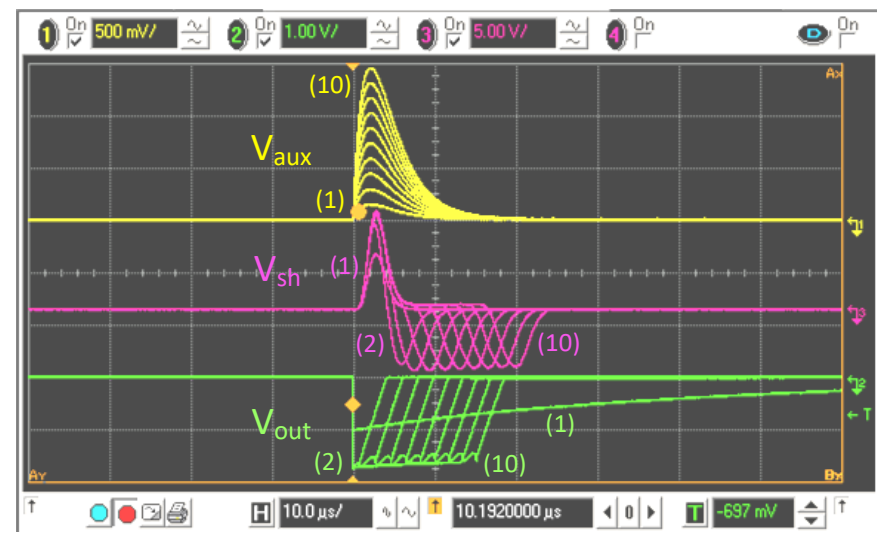

Fig. 2. Relevant signals as seen on the oscilloscope. $V_{\text {out }}$ is the preamplifier output signal as given by $\mathrm{V}_{\text {out }}-\mathrm{V}_{\text {out }} ; \mathrm{V}_{\text {sh }}$ is the signal obtained by processing $\mathrm{V}_{\text {out }}$ through a quasi-Gaussian spectroscopy amplifier. $\mathrm{V}_{\text {aux }}$ is the auxiliary signal used for high-resolution spectroscopy of the large events.

We finally measured the noise of the charge preamplifier by filtering the signal with a commercial quasi Gaussian spectroscopy amplifier as connected to $\mathrm{V}_{\text {out }}$. The detector has been simulated by connecting a capacitance of $22 \mathrm{pF}$ at the preamplifier input. We used a feedback capacitance of $1 \mathrm{pF}$. The input transistor is a BF862 operated at room temperature. An Equivalent Noise Charge of 115 electrons r.m.s. has been obtained at a shaping time of $6 \mu \mathrm{s}$ which corresponds to a pulser line width of $0.8 \mathrm{keV}$ fwhm in Ge.

Overall an exceptional spectroscopic dynamic range is obtained by using the shown technique, from $\sim 5 \mathrm{keV}$ to $\sim 180 \mathrm{MeV}$ with a non linearity of less than $\pm 0.2 \%$.

Research and development is being conducted in parallel towards the implementation of a similar technique in an Application Specific Integrated Circuit (ASIC) form [18]-[21].

\section{ACKNOWLEDGMENT}

We thank G. Pascovici and D. Bazzacco for the useful suggestions and support.

\section{REFERENCES}

[1] AGATA homepage, URL: http://www-w2k.gsi.de/agata/

[2] I. Y. Lee, "Gamma-ray tracking detectors," Nucl. Instrum. Meth., vol. A422, pp. 195-200, 1999.

[3] S. Akkoyun et al., "AGATA—Advanced GAmmaTrackingArray", Nucl. Instrum. Meth., vol. A668, pp. 26-58, 2012.

[4] A. Pullia et al., "Cross-Talk Limits of Highly Segmented Semiconductor Detectors", IEEE Trans. Nucl. Sci., vol. 58, no. 3, pp. 1201-1205, 2011.

[5] A. Pullia et al., "Characterization of HPGe-Segmented Detectors from Noise Measurements", IEEE Trans. Nucl. Sci., vol. 51, no. 6, pp. 30863089, 2004

[6] Th. Kröll et al., "In-beam experiment with the $\gamma$-ray tracking detector MARS", Nucl. Instrum. and Meth., vol. A586, pp. 421-431, 2008.

[7] Th. Kröll, D. Bazzacco, "Simulation and analysis of pulse shapes from highly segmented HPGe detectors for the gamma-ray tracking array MARS," Nucl. Instrum. Meth., vol. A463, pp. 227-249, 2001.

[8] G. Pascovici et al., "Low noise, dual gain preamplifier with built in spectroscopic pulser for highly segmented high-purity germanium detectors", WSEAS Trans. on Circuits and Systems, vol. 7, no. 6, pp. 470-481, 2008.

[9] F. Zocca et al., "A Smart Reset Circuit for Low-Noise Preamplifiers of X- $\gamma$ Ray Sensor Signals", IEEE Trans. Nucl. Sci., vol. 54, no. 1, pp. $197-$ 201, 2007.

[10] A. Pullia, G. Pascovici, C. Ur, "A versatile low-noise wide-range charge-sensitive preamplifier for HPGe detectors", 2012 IEEE Nuclear Science Symposium and Medical Imaging Conf. Rec., ISBN 978-14673-2028-3, pp. 815-818, Anaheim, CA, Oct. 27 2012-Nov. 3 2012, DOI: 10.1109/NSSMIC.2012.6551217

[11] F. Zocca, A. Pullia, D. Bazzacco, and G. Pascovici, "A Time-OverThreshold Technique for Wide Dynamic Range Gamma-Ray Spectroscopy with the AGATA Detector", IEEE Trans. Nucl. Sci., vol. 56, no. 4, pp. 2384-2391, 2009

[12] A. Pullia et al., "An Advanced Preamplifier for Highly Segmented Germanium Detectors", IEEE Trans. Nucl. Sci., vol. 53, no. 5, pp. 28692875,2006

[13] A. Pullia et al., "Extending the dynamic range of nuclear pulse spectrometers", Rev. Sci. Instrum., vol. 79, p. 036105-01, 2008, DOI: 10.1063/1.2894305

[14] A. Pullia, D. Barrientos, D. Bazzacco, M. Bellato, D. Bortolato, R. Isocrate, "A 12-channel 14/16-bit 100/125-MS/s digitizer with 24-Gb/s optical output for AGATA/GALILEO”, 2012 IEEE Nuclear Science Symposium and Medical Imaging Conf. Rec., ISBN 978-1-4673-2028-3, pp. 819-823, Anaheim, CA, Oct. 272012 - Nov. 3 2012, DOI: 10.1109/NSSMIC.2012.6551218

[15] D. Barrientos et al, "Multiple register synchronization with a high-speed serial link using the aurora protocol", IEEE Trans. Nucl. Sci., vol. 60, no. 5, pp. 3521-3525, 2013, Article number 6583338, DOI: 10.1109/TNS.2013.2273369

[16] D. Barrientos et al., "Performance of the Fully Digital FPGA-Based Front-End Electronics for the GALILEO Array", IEEE Trans. Nucl. Sci., in press, 2015, DOI: 10.1109/TNS.2015.2480243

[17] A. Pullia, G. Bertuccio, "Resolution limits of silicon detectors and electronics for soft X-ray spectroscopy at non cryogenic temperatures", Nucl. Instrum. and Meth., vol. A380, pp. 1-5, 1996

[18] A. Pullia et al, "Cryogenic Performance of a Low-Noise JFET-CMOS Preamplifier for HPGe Detectors", IEEE Trans. Nucl. Sci., vol. 57, no. 2, pp. 737-742, 2010

[19] A. Pullia, S. Capra, "Design of a resistorless ASIC preamplifier for HPGe detectors with non-linear pole/zero cancellation and controlled fast-reset feature", 2012 IEEE Nuclear Science Symposium and Medical Imaging Conf. Rec., ISBN 978-1-4673-2028-3, pp. 86-90 , Anaheim, CA, Oct. 272012 - Nov. 3 2012, DOI: 10.1109/NSSMIC.2012.6551066

[20] A. Pullia, F. Zocca, S. Capra, "An integrated low-noise charge-sensitive preamplifier with virtually unlimited spectroscopic dynamic range", 2012 IEEE Nuclear Science Symposium and Medical Imaging Conf. Rec., ISBN 978-1-4673-2028-3, pp. 693-696 , Anaheim, CA, Oct. 27 2012 - Nov. 3 2012, DOI: 10.1109/NSSMIC.2012.6551194

[21] R.J Aliaga, V. Herrero-Bosch, S. Capra, et al., "Conceptual design of the TRACE detector readout using a compact, dead time-less analog memory ASIC", Nucl. Instrum. Meth., vol. A800, pp. 34-39, November 2015, DOI: 10.1016/j.nima.2015.07.067 